\title{
Il Diritto Internazionale Può Scongiurare LE GUERRE?*
}

Livio Perra**

\begin{abstract}
1 Introduzione. 2 Gli strumenti del diritto internazionale. 3 La Lega delle Nazioni. 4 La Carta delle Nazioni Unite. 5 Il problema della sovranità nazionale. 6 Le maggioranze. 7 Le sanzioni e le misure. 8 I poteri coercitivi e l'uso della forza dell'Organizzazione delle Nazioni Unite. 9 Le operazioni di peace-keeping 10 La deroga al divieto dell'uso della forza per legittima difesa e la guerra giusta. 11 La pace giusta e il diritto dei popoli alla pace. 12 La cultura della pace. 13 Conclusioni. Bibliografia.
\end{abstract}

\section{RIASSUNTO}

Nel presente articolo l'autore muove la sua analisi partendo dall'interrogativo se il diritto internazionale possa scongiurare le guerre. Il diritto internazionale ha sempre ricercato l'obiettivo di dirimere le controversie tra gli Stati in maniera pacifica. La spinta verso questo fine si avverte in misura maggiore dopo i grandi conflitti bellici. La Lega delle Nazioni vede la propria nascita in funzione di questo intento pacificatore. Anche se viene definita come una impresa fallimentare, l'autore ritiene che abbia posto le basi per i successivi progressi. Importanti passi vengono compiuti dall'Organizzazione delle Nazioni Unite, la quale cerca di salvaguardare la pace attraverso l'utilizzo di misure che contemplano l'uso della forza e misure che non prevedono l'utilizzo della forza. In particolare, l'autore esamina entrambe le tipologie di strumenti e giunge alla conclusione che anche questo approccio sembra non essere del tutto efficace. Egli esamina il concetto di pace giusta ed il diritto dei popoli alla pace. Infine, l'attenzione si concentra sulla tendenza più recente alla promozione dell'educazione alla cultura della pace.

Parole chiave: Pace. Diritto Internazionale. Diritto dei popoli alla pace. Cultura della pace.

\section{INTRODUZIONE}

* A Revista Opinião Jurídica agradece ao Sr. José Ilo Mota, responsável pela revisão da língua italiana.

** Diplomado en Defensa Internacional de los Derechos Humanos per la escuela de Prática Jurídica de la Universidad de Zaragoza y Centro Latinoamericano de Derechos Humanos. Cultore dela matéria (Diritto Privato, IUS/01) per la Università degli Studi de Caligari. Specializzazione in Professione Legale, indirizzo Giudiziario - Forense per la Università degli Studi de Cagliari. Praticante avvocato abilitato ad esercitare il patrocínio nanti ai Tribunali del distretto dela Corte d'Appello di Caligari, limitadamente ai procedimenti de cui all 'art. 7 della legge 16.12.1999 n. 479 della Ordine degli Avvocati di Cagliari. Laurea Specialistica in Giurisprudenza per la Facoltà di Giurisprudenza, Università degli Studi de Cagliari. Professore a Contratto di Diritto del Lavoro, presso 1'Università degli Studi di Sassari. Profesore a Contratto di Istituzioni di Dirito Penale e Legislazione Minorile, presso l’ Università degli Studi di Sassari. E-mail: <livio.perra@tiscali.it〉. https://orcid. org/0000-0002-1045-1206 
Sin dalla fine della seconda Guerra Mondiale si è avuto il timore di ogni nuova guerra. Si pensi ai conflitti nei Balcani, visti con preoccupazione in Italia e spesso definiti come la "guerra vicina". Ancor oggi le notizie nei giornali di determinati fatti o focolai di guerra rievocano i ricordi dei due grandi conflitti mondiali del ventesimo secolo e provocano inquietudine all'idea di una vera e propria terza guerra mondiale.

Nel presente lavoro si analizzerà la possibilità del diritto internazionale di evitare o arginare sul nascere ogni guerra e il suo diffondersi su scala globale o l'attenuazione degli strumenti di morte e distruzione cruenti e dei danni ai civili.

I primi discorsi sulla pace nei tempi moderni arrivano all'alba della Dichiarazione Universale dei diritti dell'uomo del 1948, cioè dopo la seconda guerra mondiale. Non vi è dubbio, infatti, che il valore più sentito nell'immediato secondo dopoguerra è la pace. Troppo forte era il ricordo doloroso delle devastazioni e degli orrori. L'arma atomica aveva scosso la vita di tutti: nelle persone ha iniziato a serpeggiare il timore che la guerra con questi nuovi armamenti potesse determinare addirittura la fine della civiltà.

\section{GLI STRUMENTI DEL DIRITTO INTERNAZIONALE}

Il diritto internazionale sin dalle sue origini ha sempre cercato soluzioni alternative ai conflitti tra gli Stati per risolvere i dissidi tra essi.

Nel periodo delle monarchie assolute era il sovrano ad impegnarsi personalmente negli accordi con altri sovrani per la non belligeranza. Il rispetto di tali patti era spesso garantito dal senso dell'onore del regnante stesso, ${ }^{1}$ che sarebbe andato perso dall'infrangersi del trattato stipulato.

Va, senza dubbio, evocata la figura della nobile categoria dei diplomatici, di storica memoria, i quali mediavano per la conclusione di accordi tra le nazioni. Essi hanno prestato la loro minuziosa attività per mantenere la pace tra i popoli.

Con la seconda guerra mondiale si è assistito alla "crisi del diritto internazionale": nazioni che si erano dichiarate neutrali come il Belgio, l'Olanda, la Norvegia, la Danimarca e la Grecia sono state oggetto di attacco ed invasione senza complimenti.

Nello scenario post bellico gli Stati hanno avvertito l'esigenza di collaborare alla pace comune, con la fondazione dell'Organizzazione delle Nazioni Unite nel 1945. Vi è un precedente storico importante: l'istituzione della Lega delle Nazioni con il trattato di pace del 1919.

\section{LA LEGA DELLE NAZIONI}

Nel contesto del trattato di Versailles del 1919, con cui si sancisce ufficialmente la fine della prima guerra mondiale, gli Stati riuniti nella conferenza di pace si pongono l'obiettivo ambizioso di trovare una soluzione per impedire che un conflitto di tali dimensioni si 
ripeta. Tra i vari uomini che rappresentano gli Stati vi sono il primo ministro del Regno Unito, David Lloyd George, il presidente del Consiglio dei ministri francese Georges Benjamin Clemenceau, il presidente degli Stati Uniti d'America Thomas Woodrow Wilson e il presidente del Consiglio dei ministri italiano Vittorio Emanuele Orlando. In questa conferenza di pace si forma l'idea di addivenire al Patto della Società delle Nazioni. Vi sono varie idee, che vanno contemperate, portate dai vari rappresentanti dei singoli Stati. Wilson si fa promotore della Lega delle Nazioni e cerca di portare all'interno di questo patto i quattordici punti che aveva formulato in vista della conferenza di pace di Parigi qualche tempo prima. L'obiettivo del Patto della Società delle Nazioni è quello di istituire un organismo internazionale che sia capace di dirimere ogni controversia che sorgerà tra gli Stati in maniera pacifica, evitando i conflitti con due strumenti importanti: la soluzione diplomatica delle controversie ed il controllo degli armamenti. Il fine comune da perseguire è comunemente inteso nel rispetto dell'assetto politico territoriale stabilito nei trattati di pace. Wilson predica il principio di autodeterminazione dei popoli e, per assicurare il carattere di universalità, insiste perché lo statuto della Lega delle Nazioni sia incluso nel Trattato di Pace, esplicitando in tal modo l'intento di far divenire l'accettazione dei due indivisibile.

Il carattere universale favorisce la presenza di quasi tutte le nazioni del mondo in maniera tale da scongiurare ogni possibile conflitto, se non fosse per il fatto che concretamente gli Stati Uniti d'America non ratificano il patto, inoltre è assente la Germania in quanto sconfitta e con interessi confliggenti rispetto ad alcuni di quelli in suo danno degli Stati vincitori che sono trasfusi nel patto, e nemmeno la Russia è presente. Orlando mette in discussione nel suo scritto La crisi del Diritto internazionale il carattere di universalità osservando la mancanza proprio di questi tre Stati. Inoltre, l'autorità di questo nuovo "ordinamento internazionale" è del tutto compromessa dal fatto della mutabilità notevole dell'estensione territoriale: con troppa facilità si è ammessi o ci si dimette dalla Lega delle Nazioni. Wilson si fa paladino della sovranità degli Stati, dell'uguaglianza e della parità di ciascun membro anche in termini di peso del voto di ciascuno di essi nelle decisioni prese a maggioranza. Altro nodo che lascia perplessi è la pregiudiziale negativa britannica, secondo cui una nazione non si può vincolare ad un obbligo derivante da una decisione presa se contraria alla propria volontà. All'interrogativo di Orlando, come egli stesso riporta, sul modo di superare l'insuperabile pregiudiziale negativa, Wilson risponde con "una commovente fiducia: l'opinione pubblica del mondo" (ORLANDO, 2014, p. 22). A Wilson ${ }^{2}$ va riconosciuta l'ammirevole idea di voler sempre garantire l'indipendenza politica e territoriale di tutti gli Stati anche quelli più piccoli.

La Società delle Nazioni viene estinta nel 1946 dopo il fallimento manifestatosi con la seconda guerra mondiale. Nonostante la Società delle Nazioni sia considerata un fallimento, lascia un'importante eredità per gli enti internazionali che saranno istituiti nell'avvenire con l'intento di portare la pace nel mondo: il carattere dell'universalità e la rappresentanza di tutti gli Stati grandi o piccoli che siano. 


\section{LA CARTA DELLE NAZIONI UNITE}

Nella Conferenza di San Francisco (25-26 giugno 1945) è adottata la Carta delle Nazioni Unite. Questo trattato istituisce l'Organizzazione delle Nazioni Unite. Gli intenti delle Nazioni Unite che si leggono già nel preambolo della Carta (o Statuto), sono quelli di "salvare le future generazioni dal flagello della guerra" (CARTA..., 1945, online), di riaffermare i diritti fondamentali, di creare le condizioni per il rispetto degli obblighi derivanti dal diritto internazionale e di promuovere il progresso sociale.

Al fine della pace, sono dedicate numerose disposizioni del Trattato istitutivo delle Nazioni Unite.

Nell'articolo $1^{\circ}$ si fa preciso riferimento tra i fini alla pace e alla sicurezza internazionale come ad un qualcosa da preservare. Si fa spesso riferimento, non solo nell'articolo $1^{\circ}$, allo sviluppo egualitario di relazioni amichevoli tra gli Stati. (CARTA..., 1945).

Gli Stati Membri devono ottemperare agli obblighi del trattato, in particolare devono ricercare soluzioni pacifiche per dirimere le controversie internazionali ed evitare di mettere in pericolo la pace. Per risolvere le controversie tra gli Stati può essere adita la Corte Internazionale di Giustizia, come precisato nell'articolo 38 del proprio Statuto, la quale decide, oltre che conformemente alle convenzioni internazionali riconosciute dai Paesi in lite, alla consuetudine internazionale ed ai principi generali di diritto riconosciuti dalle nazioni civili, in base al diritto internazionale ex aequo et bono nei casi in cui le parti siano d'accordo. La Corte Internazionale di Giustizia è l'organo giurisdizionale delle Nazioni Unite (articolo 92 della Carta delle Nazioni Unite), essa ha inoltre il potere di emettere pareri su richiesta dell'Assemblea Generale, del Consiglio di Sicurezza e di altri enti autorizzati a richiederli dall'assemblea (articolo 96 della Carta delle Nazioni Unite). Si tratta di pareri facoltativi e non vincolanti, ma sicuramente sono pur sempre pareri autorevoli (CARTA..., 1945).

Vi è il divieto dell'uso della forza nelle relazioni internazionali, codificato all'articolo $2^{\circ}$ della Carta delle Nazioni Unite. L'unica eccezione all'uso della forza si trova nell'articolo 51 della Carta e si riferisce al diritto naturale di autodifesa individuale o collettiva quando uno Stato subisca un attacco armato. Entrambe queste disposizioni sono, inoltre, considerate norme internazionali di ius cogens e quindi hanno carattere imperativo e generale (CARTA..., 1945).

\section{IL PROBLEMA DELLA SOVRANITÀ NAZIONALE}

Il problema della sovranità degli Stati irrompe sicuro quando si parla di trattati internazionali, a volte in maniera lieve, a volte in maniera più energica.

La questione concerne il quanto uno Stato è disposto a cedere o a rinunciare alla propria sovranità e la misura con la quale esso si vincoli.

Nella Lega delle Nazioni del 1919 si è cercato di preservare quanto più possibile la sovranità delle nazioni e questo non ha favorito di certo il concretizzarsi dei migliori intenti, 
provocando una situazione di incertezza, potendo ogni Stato uscirvi con facilità. In particolare, come scrive Orlando (2014, p. 19):

Si potrebbe definire la Lega delle Nazioni come un colossale esperimento di laboratorio, che sarebbe stato diretto alla creazione di un vero e proprio ordinamento giuridico internazionale, che doveva assicurare o, quanto meno, dare delle garanzie contro l'arbitrario imperialistico interesse di guerre: e ciò [...] senza compromettere il principio della sovranità ed eguaglianza degli Stati.

Anche nella carta delle Nazioni Unite si pone il problema della sovranità dei singoli Stati. Le Nazioni Unite in linea di massima garantiscono la sovranità degli Stati membri (nel senso della non ingerenza nelle questioni interne degli Stati). In certi casi il divieto di ingerenza diventa più flessibile o meglio si distende per l'inserimento di garanzie giurisdizionali contro le violazioni della pace e dei diritti umani, come avviene ad esempio nel ruolo complementare alle Corti penali nazionali della Corte Penale Internazionale. In particolare, poi, la Carta delle Nazioni Unite intacca la sovranità nazionale degli Stati con due principi fondamentali quali la preservazione della pace e della sicurezza internazionale e la tutela dei diritti umani (PASTORE, 2001, p. 167).

L'interrogativo su quanto uno Stato possa rinunciare alla propria sovranità per dei "fini superiori" è sempre stato argomento di discussioni sopraffini tra i giuristi ed i filosofi.

Si pensi all'idea di Immanuel Kant, come ricorda anche Bobbio (2003, p. 565). Kant molto probabilmente è influenzato dalla lettura dei testi di Thomas Hobbes. In particolare, egli ritiene che, per aversi una pace perpetua, gli Stati da uno stato di natura devono ascendere verso una confederazione permanente di Stati attraverso la stipula di un patto. Ma Kant rifiuta l'idea di uno Stato di Stati, per non scivolare nel dispotismo. Non mancano teorie più varie, da quelle che vedono come soluzione per la pacificazione perpetua l'istituzione di uno Stato mondiale (federazione di Stati sul modello degli Stati Uniti d'America) a quelle che sostengono, invece, l'idea di un controllo maggiore del popolo sul governo dello Stato. Infine, non mancano teorie, spesso anche religiose, che vedono come soluzione una vera e propria educazione degli individui alla pace.

\section{LE MAGGIORANZE}

Il problema delle maggioranze si pone riguardo alle decisioni degli organismi sovranazionali quando devono essere prese delle decisioni che incidono sugli Stati. La questione, cioè, è se debba prevalere la maggioranza dei componenti, se sia necessaria l'unanimità e se ci siano dei componenti che possono avere il diritto di veto sulle decisioni.

Vittorio Emanuele Orlando (2014, p. 23) osserva che la decisione in un'assemblea che delibera con la maggioranza dei voti dei suoi membri risponde ad una notevole esigenza pratica: "si può e si deve considerare come un principio accolto per un criterio di necessità, senza del quale quella pluralità non potrebbe, spesso, pervenire ad una conclusione.” Questo sistema si espone a delle perplessità. Si aprono due strade: 
considerare che vi sia un consenso iniziale presentato da ciascuno Stato con l'adesione all'organismo internazionale ad assoggettarsi ad ogni decisione presa a maggioranza dei membri, oppure la possibilità di non applicazione allo Stato dissenziente della determinazione votata in assemblea.

Vittorio Emanuele Orlando riferisce che i Romani, attenti alle questioni pratiche, avessero escogitato una singolare soluzione:

I Romani, infatti, nei casi in cui ammisero il principio maggioritario, istituirono una specie di presunzione iuris et de iure, per cui la deliberazione della maggioranza si intendeva che fosse stata voluta di tutti i votanti, anche da quelli rimasti in minoranza. Ed essi, in un campo di gran lunga più importante e più vasto, applicarono il principio per cui una pluralità di volontà richiesta per una decisione debba essere unanime, indipendentemente quindi e al di sopra del principio di maggioritario: e ciò proprio a proposito della organizzazione del massimo dei loro uffici quali fu il Consolato (ORLANDO, 2014, p. 24).

Infatti, non mancano concezioni dove l'uomo non si ritenga obbligato neppure una maggioranza ha assunto una determinata deliberazione in un'assemblea. Scrive a tal riguardo Orlando (2014, p. 24):

Contro il principio che la maggioranza obbliga la minoranza, l'uomo, in quanto libero, tende a non ritenersi impegnato senza il concorso della sua personale volontà: il che vale quanto dire che se si tratta di una deliberazione affidata ad una pluralità di persone, la condizione per una generale validità e obbligatorietà di essa consisterebbe sempre nella unanimità dei voleri.

Le Nazioni Unite, come si evince dalla Carta e dallo Statuto della Corte Internazionale di Giustizia, prediligono il principio di maggioranza ${ }^{3}$ formalmente, anche se non manca un tentativo sempre crescente di prendere decisioni maggiormente condivise. Non mancano aspetti di critiche teoriche al fatto che il voto di un piccolo Stato valga allo stesso modo di quello dei grandi Stati, ma le Nazioni Unite tendono alla rappresentazione egualitaria di ciascun proprio Stato membro.

\section{LE SANZIONI E LE MISURE}

Un problema di notevole rilevanza è quello delle sanzioni per la violazione dei patti di non belligeranza in genere, o delle disposizioni contenute nei trattati di enti internazionali a tal riguardo. In pratica la questione ruota sulla considerazione della forza coercitiva tesa all'effettivo rispetto degli accordi e dei trattati internazionali.

Neppure vero il discorso circa la giuridicità delle norme che non possiedono strumenti coercitivi che utilizzano la forza per il proprio rispetto, alcuni giuristi ritengono che le norme hanno come condizione necessaria di giuridicità (cioè una norma viene qualificata come giuridica) la coazione intesa nel senso dell'uso della forza per la propria ottemperanza. Si può ipotizzare la soluzione o il cambio di prospettiva nel problema perché come scrive Orlando (2014, p. 6): 
Gli stessi sostenitori della dottrina la quale ammette che si possa dare una norma giuridica anche se non accompagnata dalla condizione di coercibilità per mezzo della forza, riconoscono, tuttavia, che norme sprovviste di tale condizione siano da considerarsi come imperfette. Ed è questa la ragione sostanziale per cui si può affermare che questo carattere di imperfezione è universalmente riconosciuto dagli stessi autori di diritto internazionale: si disputa solo sul grado di tale imperfezione.

La dottrina e la giurisprudenza internazionale ritengono che il divieto della forza nelle relazioni internazionali, salvo il caso della legittima difesa, sia da classificare tra le disposizioni di ius cogens. Da questo consegue che, in base all'articolo 53 della Convenzione di Vienna del 1969 sul diritto dei trattati, ${ }^{4}$ non possa concludersi un trattato in contrasto o per derogare questo divieto, avendo esso il carattere di norma imperativa del diritto internazionale generale, riconosciuta ed accettata dalla comunità internazionale degli Stati, cioè di norma non derogabile e non modificabile se non con una fonte di pari grado.

Il problema si pone forte quando i trattati non vengono rispettati ${ }^{5}$ circa le sanzioni che possono essere corrisposte ed applicate.

Va considerato preliminarmente che non vi è una autorità sovraordinata o gerarchicamente superiore agli Stati ed agli enti internazionali che ha il potere di attuare coattivamente il diritto. La legittimazione all'applicazione delle sanzioni è del Consiglio di Sicurezza delle Nazioni Unite e gli Stati lesi nei propri diritti da violazioni possono adottare contromisure. Le sanzioni e le contromisure sono per lo più di natura economica. Tuttavia, il Consiglio di Sicurezza può porre in essere misure militari collettive quando uno o più Stati membri violano la pace o aggrediscono altri Stati. In particolare, l'uso della forza è considerato come extrema ratio. Le sanzioni sono una risposta, si propongono di essere un deterrente al prosieguo delle azioni "vietate", sono cioè finalizzate a far cessare la condotta illecita, non hanno carattere né afflittivo, né punitivo, per questo motivo alcuni dubitano del carattere di sanzione di queste misure. Spesso, infatti, si parla di difesa legittima o di misure attuate per la sicurezza collettiva.

Riguardo a questa natura un po' particolare dell'uso legittimo della forza, Orlando sottolinea che riguardo al problema della guerra

Se considerato nella sua forma di uso della forza, sia pure come una extrema ratio, per sorreggere e garantire uno scopo conforme al diritto, dà luogo ad una risposta puramente negativa e cioè che il diritto internazionale non dispone di una forza coattiva capace di evitare le guerre anche se formalmente dirette ad attuare sanzioni repressive di atti di guerra o conducenti ad una guerra. Che se poi l'urto bellico si verificasse, l'esito di esso non avrebbe l'immediato significato di una vittoria della difesa giuridica ma attesterebbe soltanto la prevalenza della forza di uno dei due contendenti (ORLANDO, 2014, p. 5-6). 


\section{I POTERI COERCITIVI E L'USO DELLA FORZA DELL'ORGANIZZAZIONE DELLE NAZIONI UNITE}

Le Nazioni Unite devono dare una pronta risposta nel caso sia violato l'obbligo degli Stati di astenersi nel contesto delle relazioni internazionali dalla minaccia e dall'uso della forza contro l'integrità territoriale o l'indipendenza politica di qualsiasi Stato, o incompatibilmente con i propri fini.

La Carta delle Nazioni Unite prevede sia strumenti di soluzione pacifica (per lo più strumenti conciliativi per la soluzione delle controversie internazionali), sia strumenti più incisivi come l'adozione di strumenti coercitivi.

Per prima cosa va precisato che, in base all'articolo 39 della Carta delle Nazioni Unite, il Consiglio di Sicurezza deve accertare se vi sia una minaccia o una violazione della pace, o un atto di aggressione. A questo punto il Consiglio può fare raccomandazioni agli Stati (funzione essenzialmente conciliativa) o decidere altre misure più idonee al mantenere o ristabilire la pace e la sicurezza internazionale (CARTA..., 1945).

Vi è la possibilità, espressa nell'articolo 40 della Carta, delle misure provvisorie, utilizzate per prevenire l'aggravarsi di determinate situazioni, ad esempio vanno ricordate tra queste le risoluzioni per il cessate il fuoco, la richiesta del ritiro delle truppe.

È necessario distinguere tra le misure che non implicano l'uso della forza (articolo 41) e le misure che implicano l'uso della forza (articolo 42) (CARTA..., 1945).

Le misure che non implicano l'uso della forza sono decise dal Consiglio di Sicurezza. Queste misure consistono, essenzialmente, nelle interruzioni totali o parziali delle relazioni economiche e delle comunicazioni ferroviarie, marittime, aeree, postali, telegrafiche, radio e la rottura delle relazioni diplomatiche. Ai sensi dell'articolo 50 della Carta, nei casi in cui sorgano particolari difficoltà economiche dall'applicazione di tali misure per altri Stati anche se non membri, questi possono consultare il Consiglio di Sicurezza per trovare una soluzione appropriata. Solitamente nella prassi si concretano queste soluzioni in forme di assistenza o deroghe. Sono anche possibili piccole deroghe per garantire generi alimentari e medicinali alle popolazioni dello Stato sanzionato.

All'articolo 42 della Carta sono previste le misure che contemplano l'uso della forza. Esse consistono in ogni azione che sia necessaria per mantenere o ristabilire la pace e la sicurezza internazionale e possono essere attuate con forze aeree, navali o terrestri. Per queste azioni la Carta prevede una disciplina particolare negli articoli dal 43 al 49. In particolare, gli Stati forniscono mezzi e facilitazioni (spazio aereo, diritto di passaggio) con appositi accordi speciali conclusi tra il Consiglio di Sicurezza e i singoli Stati membri. Vi è il Comitato di Stato Maggiore (composto dai capi di Stato Maggiore degli Stati Membri permanenti del Consiglio di Sicurezza, o dai loro rappresentanti) che si occupa del coordinamento dell'uso della forza e dei piani strategici. Il Comitato di Stato Maggiore dipende ed è responsabile nei confronti del Consiglio di Sicurezza. 
Le misure che prevedono l'uso della forza possono essere esperite quando vengano ritenute inadatte quelle misure "pacifiche" anche se non sono state applicate precedentemente.

\section{LE OPERAZIONI DI PEACE-KEEPING}

Con il termine peace-keeping si intendono quelle operazioni non contemplate nell'articolo 42 della Carta delle Nazioni Unite, ma nate nella prassi delle Nazioni Unite. Esse assumono carattere imparziale e hanno funzioni umanitarie, come l'invio e la distribuzione di aiuti ai civili ed i compiti di prevenzione per scongiurare l'insorgere dei conflitti. Queste operazioni non hanno affatto carattere coercitivo e si ricorre all'uso della forza solo ed esclusivamente per legittima difesa. Gli Stati volontariamente si impegnano con appositi accordi e caso per caso a conferire alle Nazioni Unite truppe per questo scopo. Inoltre, è necessario il consenso delle parti ostili, perché si possa procedere a questa tipologia di intervento. Questo si comprende alla luce del rispetto della non ingerenza nelle questioni degli altri Paesi.

$\mathrm{Al}$ fine di legittimare queste operazioni giuridicamente sono sorte numerose teorie della dottrina.

Alcuni autori fanno rientrare queste operazioni nelle misure provvisorie dell'articolo 40 della Carta delle Nazioni Unite. Altri autori fanno rientrare queste operazioni tra quelle che contemplano l'uso della forza dell'articolo 42 della Carta, ma come sottolinea Santini (CAPECCHI; PARISI; SANTINI, 2002, p. 30) sarebbe una qualificazione corretta nei casi in cui vi sia anche un mandato allargato al peace-enforcement. Altri ancora poggiano la giustificazione di queste operazioni sulla teoria dei poteri impliciti, o sulla base del conferimento degli Stati membri al Consiglio di Sicurezza della responsabilità principale del mantenimento della pace e della sicurezza internazionale per assicurare un'azione pronta ed efficace delle Nazioni Unite (come previsto nell'articolo 24 della Carta delle Nazioni Unite) (CARTA..., 1945).

\section{LA DEROGA AL DIVIETO DELL'USO DELLA FORZA PER LEGITTIMA DIFESA E LA GUERRA GIUSTA}

La legittima difesa consiste in uno strumento di autotutela individuale o collettiva quando uno Stato membro sia sotto attacco armato. L'articolo 51 della Carta delle Nazioni Unite riconosce la legittima difesa come diritto naturale che lo stesso Statuto non pregiudica. Dunque, la legittima difesa è consentita finché il Consiglio di Sicurezza prenda le misure necessarie per mantenere la pace e la sicurezza internazionale. Tali misure devono essere portate a conoscenza del Consiglio di Sicurezza, il quale può intraprendere le azioni per mantenere o ristabilire la pace e la sicurezza internazionale (CARTA..., 1945).

La legittima difesa non pone particolari problemi circa la sua presenza, le questioni maggiori si pongono per cosa sia da intendere come legittima difesa. Se senza dubbio la 
legittima difesa opera nel caso di un attacco allo Stato che la invoca, pare più difficilmente concretarsi nei casi di legittima difesa preventiva ipotizzati da alcuni Stati ${ }^{6}$ o addirittura allargati così tanto da voler ritenere legittima difesa anche l'intervento per salvaguardare i propri cittadini in pericolo di vita in altri Stati. Non v'è dubbio che la legittima difesa debba avere il carattere della necessità, dell'immediatezza e della proporzionalità e nel caso sia legittima difesa collettiva debba esserci la richiesta o il consenso dello Stato attaccato all'intervento di un altro Stato.

Sull'uso della forza legittimo o sulla guerra giusta è stato scritto un mare di inchiostro da parte di giuristi, filosofi e teorici di politica.

Particolari sono le parole di Viola (2005) per una completa riflessione sulla guerra giusta. Egli afferma che è molto importante che si riprendano ai giorni nostri i discorsi sulla guerra giusta, perché oggi si suole parlare di pace giusta e di conseguenza i mezzi per preservarla diventano "giusti". La teoria della guerra giusta può essere rifiutata dalle teorie pacifiste che condannano ogni forma di guerra anche nel caso dell'autodifesa.

Secondo il diritto naturale viene qualificato come giusto il diritto di difendersi dall'aggressore, quindi è giusta la guerra di difesa perché serve per proteggere i beni dello Stato, per il pagamento di un debito o punire i crimini di un nemico pubblico, queste concezioni rientrano nel concetto di difesa nazionale che spetta allo Stato. ${ }^{7}$ Con il tempo il concetto di autodifesa è andato a comprendere i più vari contenuti, fino a giungere all'opinione attuale quasi sempre condivisa di essere legittima a determinate condizioni quali l'esistenza di un pericolo non presunto, ma certo e concreto.

Secondo la teoria di Tommaso d'Aquino ${ }^{8}$ perché la guerra sia giusta non basta il requisito dell'autodifesa: egli parla del concetto di bene comune e della esigenza della sua preservazione. Gli influssi di questa teoria possono scorgersi nelle misure che contemplano l'uso della forza per mantenere il "bene comune" della pace e della sicurezza internazionale.

Altra tipologia di "guerra giusta" che va affermandosi nella prassi ai giorni nostri, fuori dalle previsioni della Carta delle Nazioni Unite, ma con forti richiami alla visione della guerra come giusta in senso agostiniano della difesa del più debole e dell'oppresso, sono le guerre in difesa dei diritti umani e quelle che si pongono l'obiettivo di rimuovere le tirannie. È da tener conto che questa tipologia di guerra "giusta" può sconfinare nell'ingerenza nelle politiche nazionali altrui, ogniqualvolta la guerra sia lo strumento con cui ci si prefigga lo scopo di imporre i propri valori in terra straniera. ${ }^{9}$

Il problema si pone in maniera più decisa circa la possibilità di intervenire in uno Stato per abusi dello stesso nei confronti dei propri cittadini, perché tali ipotesi non sono contemplate nella Carta delle Nazioni Unite. A questo riguardo, parrebbe fondarsi una causa ulteriore di guerra giusta accanto a quella prevista per l'autodifesa, cioè quella per la tutela dei diritti umani, ma queste due forme spesso rischiano addirittura di confliggere tra esse.

Nella guerra per la difesa dei diritti umani la causa dovrebbe essere giusta perché si oppone ad una generalizzata violazione della dignità umana. Il carattere di giusta 
deriverebbe, poi, dall'essere decretata da una legittimata autorità internazionale con modalità proporzionate al male da abolire. In linea teorica, si aprono numerosi interrogativi di non facile soluzione: la questione sui principi, quella sulla qualificazione della guerra, quella sui modi. Si osserva sul punto dei principi che non c'è un'unica concezione di diritti umani e il giudicare la presenza o meno di una violazione potrebbe non trovare sempre un'apprezzabile condivisione. Sembra difficile qualificare un simile intervento deliberato da un organismo internazionale come guerra. ${ }^{10} \mathrm{Vi}$ è poi la questione che riguarda la possibilità di "violare i diritti umani per difendere i diritti umani”. Ogni guerra è caratterizzata dalla violazione dei diritti degli individui, la natura stessa della guerra lo implica. Va da sé che una guerra giusta diventa ingiusta se sono utilizzati mezzi ingiusti. Così, questo terzo aspetto apre un paradosso: la guerra violando i diritti è di conseguenza sempre contraria al diritto e difficilmente qualificabile come giusta. ${ }^{11}$

\section{LA PACE GIUSTA E IL DIRITTO DEI POPOLI ALLA PACE}

La pace è il principale fine della comunità internazionale. Il valore della pace è in continua evoluzione. Inizialmente veniva inteso sotto una concezione negativa: l'assenza della guerra. Nella visione di Bobbio (1998, p. 165) (VIOLA, 2005, 46-47), oltre alla concezione "negativa" di pace è presente anche una "positiva" propria del diritto internazionale: la regolamentazione che mette un punto sulla guerra tra Stati con accordi e trattati di pace. Questo è un discorso tecnico giuridico e non si può attribuire il carattere di giustizia o ingiustizia alla pace. Secondo Viola (2005, p. 47), più di cosa sia la pace si discute oggi di cosa dovrebbe essere ricondotto al contenuto del termine pace. Il concetto di pace, se si considerano i beni che vi si possono ricomprendere, sarebbe vasto e indeterminato, pertanto per comprendere il concetto giuridico di pace bisogna far riferimento a quello indicato nei testi giuridici e nelle interpretazioni che ne vengono date. In questo modo, seguendo questo suggerimento di metodo, bisogna ora osservare l'idea di pace contenuta nella Carta delle Nazioni Unite e le sue interpretazioni. Nella Carta si deve volgere lo sguardo al concetto espresso di "mantenimento della pace e della sicurezza" ed alle interpretazioni che ne sono state date.

La prima linea interpretativa si riferisce alla fine della seconda guerra mondiale, al mantenimento della pace intesa come cessazione delle ostilità e del conflitto e al porre in essere l'eliminazione delle cause che hanno portato alla guerra. ${ }^{12}$ Allo scopo di mantenere questa situazione di non guerra si privilegia la soluzione pacifica alle controversie internazionali e si ripudia la guerra, si punta, anche, sul principio di autodeterminazione dei popoli per eliminare gli ultimi fenomeni di colonialismo, ritenuti una delle cause del secondo conflitto mondiale.

La concezione della conservazione dello stato di non belligeranza è rimasta durante tutta la guerra fredda e la Carta delle Nazioni Unite in quegli anni ha garantito l'equilibrio internazionale. Inoltre, si trattava di un gioco di equilibri, in cui le dimostrazioni della 
forza potenziale erano sufficienti ad evitare un possibile scontro. Attualmente bisogna osservare che vi sono nuovi parametri di valori per i quali non ci si può limitare al semplice mantenimento della pace e della sicurezza. Inoltre, il valore della pace sta prendendo le distanze contenutistiche da quello della semplice sicurezza. Valori nuovi e nuove riletture degli stessi portano il concetto di pace ad avvicinarsi sempre più a quello di giustizia. Le Nazioni Unite non intendono solo garantire la gestione non violenta dei contrasti internazionali da parte degli Stati. Il concetto di pace professato si arricchisce di contenuti alla luce delle nuove esigenze che si affacciano su scala globale. È una pace che esige per prima cosa il rispetto, la tutela e la protezione dei diritti umani. Questa è un'interpretazione che sorge dalla lettura orientata al principio di giustizia (articolo 1 della Carta delle Nazioni Unite) e dal rispetto dei diritti umani (articolo 55 della Carta delle Nazioni Unite). La pace che attualmente viene predicata, ricercata e sperata non è più una semplice assenza di guerra, ma la pace giusta. Si ritiene, infatti, che se non è giusta, la pace non è reale, ma solo un qualcosa di apparente. Si tratta, dunque, di una pace qualificata come giusta, che tiene in considerazione l'esigenza del rispetto dei diritti umani. ${ }^{13}$

A questa nuova concezione che collega la pace ai diritti umani vi è da aggiungere che la stessa pace viene sempre più affermata come diritto. In particolare, l'Assemblea Generale delle Nazioni Unite con la Risoluzione 39/11 del 12 novembre 1984 ha approvato la Dichiarazione sul diritto dei popoli alla pace. Le Nazioni Unite hanno dato un forte segnale, anche se non è stato colto da tutti gli Stati.

La forza con cui viene affermato il diritto alla pace in questa dichiarazione è notevole: si parla della pace come "sacro diritto" dei popoli della Terra. La dichiarazione prevede che gli Stati hanno l'obbligo fondamentale di salvaguardare e promuovere la pace. Essi devono attuare strumenti per la composizione pacifica delle controversie internazionali, indirizzare le proprie politiche per evitare le guerre ed in particolare le guerre nucleari e più in generale per rinunciare all'uso della forza. Poi la dichiarazione invita gli Stati e le organizzazioni internazionali ad adottare misure nazionali e internazionali per realizzare il diritto dei popoli alla pace.

In questa dichiarazione le Nazioni Unite ribadiscono i principi e i fini della salvaguardia e promozione della pace più volte ribaditi nel proprio Statuto. Si fanno portavoce del desiderio dei popoli di estirpare la guerra e evitare catastrofi nucleari affinché la pace duratura proprio in quest'epoca possa contribuire al miglioramento ed alla conservazione della vita e della civiltà sul nostro pianeta.

\section{LA CULTURA DELLA PACE}

Molte sono le teorie che ritengono che si debba educare alla pace, che si debba scoprire o riscoprire la cultura della pace.

Non mancano tra queste le teorie religiose che sostengono il valore della pace, come ad esempio il cristianesimo che ha influenzato nei secoli i discorsi sulla pace e sulla guerra. 
Vanno sottolineati i discorsi sulla pace di vari capi religiosi, dal Papa al Dalai Lama, alle riunioni, ai concili interreligiosi per promuovere la cooperazione e tendere verso la direzione della pace globale. Queste teorie non sono solo proprie della religione, ma hanno una diffusione più ampia. Si pensi alle teorie pacifiche di alcuni leader politici come Mahatma Gandhi. Si pensi alle rivendicazioni dei popoli indigeni in America Latina, dove vengono riscoperti i valori di vivere in pace e armonia con tutti gli esseri viventi e con la natura. Si pensi, inoltre, alle varie iniziative che vengono proposte nelle scuole per educare alla pace. Le Nazioni Unite non potevano non considerare che l'educazione alla cultura della pace è uno strumento capace di rafforzare la concezione collettiva di rifiuto della guerra e di una vita armoniosa e pacifica degli individui e dei popoli.

Le Nazioni Unite, richiamando i principi della Carta, la Dichiarazione Universale dei diritti dell'uomo, la Convenzione di Londra 16 novembre 1945 per la creazione dell'Organizzazione delle Nazioni Unite per l'educazione, la scienza e la cultura (UNESCO) che afferma "le guerre nascono nello spirito degli uomini, è nello spirito degli uomini che devono essere elevate le difese della pace", ricordando la propria Risoluzione 52/15 del 20 novembre 1997 che proclama l'anno 2000 "Anno internazionale della cultura della pace" e la propria Risoluzione 53/25 del 10 novembre 1998 che proclama il periodo 2001-2010 come il "Decennio internazionale della promozione di una cultura della nonviolenza e della pace a beneficio dei bambini del mondo", puntano sulla cultura e sull'educazione alla pace e proclamano la Dichiarazione su una cultura della pace con la Risoluzione 53/243 del 6 ottobre 1999.

Questa dichiarazione si rivolge ai governi, alle organizzazioni internazionali e alla società civile e li invita ad ispirarsi a quanto contiene per promuovere la cultura della pace.

All'articolo 1º della dichiarazione le Nazioni Unite spiegano cosa intendono per "cultura della pace". Essa è l'insieme di valori, di attitudini, di tradizioni, di comportamenti e di stili di vita. Questi devono tendere al rispetto della vita, della sovranità, dell'integrità territoriale e dell'indipendenza degli Stati e alla non ingerenza. Inoltre, è importante rispettare i diritti umani e le libertà fondamentali. Si deve promuovere la soluzione pacifica dei conflitti, il diritto allo sviluppo, la parità di diritti tra uomo e donna, il rispetto della libertà di espressione, d'opinione e d'informazione. È necessario conformarsi ai principi di libertà, giustizia, democrazia, tolleranza, solidarietà, rispettare il pluralismo della diversità culturale, incoraggiare lo sviluppo nazionale e internazionale che favorisce la pace. Secondo la Dichiarazione bisogna trasformare i valori, favorire i modelli di vita e seguendo questi principi favorire la pace tra gli individui e le nazioni.

Di conseguenza questi principi portano con sé dei corollari, cioè le Nazioni Unite ritengono che per la cultura della pace siano necessari anche il rispetto della soluzione pacifica dei conflitti, il rispetto delle obbligazioni derivanti dalla Carta delle Nazioni Unite e più in generale del diritto internazionale. Si rende necessario promuovere la democrazia e la partecipazione, lo sviluppo universale e i diritti umani.

Si deve, pertanto, favorire il dialogo e la mediazione, estirpare la povertà, l'analfabetismo 
ed ogni forma di discriminazione, razzismo o xenofobia. Occorre promuovere e rispettare i diritti dell'infanzia, il diritto all'informazione, la tolleranza e la solidarietà verso tutte le culture, etnie e religioni. È data notevole importanza anche al favorire il diritto di autodeterminazione dei popoli.

La cultura della pace deve essere promossa e rinforzata dai governi, dalla società civile, dai media e da tutti coloro che giocano un ruolo importante nell'educazione (insegnanti, gruppi religiosi, chi svolge attività scientifica, artistica). Ciascuno nel proprio campo deve promuovere la cultura della pace.

Una disposizione importante in chiusura si trova nell'articolo 9 e prevede che l'Organizzazione delle Nazioni Unite continui ad esercitare un ruolo decisivo per la promozione e il consolidamento della cultura della pace nel mondo.

A questa dichiarazione è allegato il programma d'azione per la cultura della pace, che prevede i fini, le strategie ed i principali attori, nonché le misure per rinforzare le attività degli attori nazionali, regionali e internazionali.

\section{CONCLUSIONI}

Nel presente articolo si è analizzata la questione se il diritto internazionale possa scongiurare le guerre. Dopo gli orrori dei due conflitti mondiali, gli Stati hanno cercato di evitare il ripetersi di tante atrocità. Il problema da subito rilevato è la mancanza di un'autorità sovraordinata agli Stati che possa irrogare sanzioni per l'inosservanza dei trattati internazionali. Le Nazioni Unite si sono adoperate sin dalla loro fondazione per la difesa dei diritti umani e si sono poste l'obiettivo di mantenere la pace nel mondo. Le misure e le sanzioni adottate su decisione del Consiglio di Sicurezza delle Nazioni Unite, da quelle di natura economica all'uso legittimo della forza verso chi minaccia la pace, spesso si pongono come strumento per far cessare determinate condotte, ma per prevenire le guerre o stroncarle sul nascere gli strumenti sembrano poco efficaci nella prassi. È difficile parlare di pace singolarmente, essendo la pace collegata o conseguente ad una guerra precedente. Ma si è visto come negli ultimi tempi il concetto di pace come "non guerra" stia lasciando il posto alla "pace giusta" ed al diritto dei popoli alla pace.

Non bisogna sottovalutare il grande contributo delle Nazioni Unite nel cercare di dirimere le controversie tra gli Stati in maniera pacifica. L'uso legittimo della forza è sempre da intendersi come extrema ratio. Notevole è, inoltre, l'evoluzione del diritto internazionale soprattutto negli ultimi settant'anni. L'idea delle prime teorizzazioni in materia dove si pensava che uno Stato si sentisse vincolato ai trattati internazionali per il fatto di essersi impegnato è stata smentita con il tempo e in particolare con la seconda guerra mondiale, dove lo stato di neutralità di alcune nazioni non è stato rispettato, dove spesso è stato disatteso lo ius ad bellum, consolidatosi nei secoli, e la volontà di alcuni governanti è andata contro la parola data negli accordi con altri Stati. Molti hanno ritenuto che per ovviare al mancato rispetto dei trattati internazionali sarebbe opportuno avere una forte autorità centrale che possa irrogare sanzioni per la violazione degli stessi, nella misura in cui nei singoli Stati vengono 
corrisposte le sanzioni per violazioni del diritto interno. ${ }^{14}$ Il fatto della mancanza di questo tipo di sanzioni ha fatto addirittura ritenere ad alcuni che non ci si trovi dinnanzi a norme giuridiche, ma è stato giustificato da altri con l'assunto che siano norme vincolanti tra gli Stati che aderiscono ad una determinata convenzione o che ratificano uno specifico trattato. Alcuni hanno ipotizzato che la soluzione sia l'opposto, cioè far partecipare in maniera maggiore il popolo alle decisioni dello Stato. Altri ancora ritengono che si debba provvedere all'educazione alla pace degli individui. In particolare nella Dichiarazione su una cultura della pace delle Nazioni Unite riveste un ruolo importante l'educazione alla pace ad ogni livello, dalla scuola ai gruppi religiosi, dallo Stato alle organizzazioni internazionali, dall'arte ai mass media. Si fa riferimento alla pace che può essere raggiunta educando alla tolleranza, al rispetto delle culture diverse, alla solidarietà e allo sviluppo universale, al rispetto dei diritti umani. Oltre a ciò, le Nazioni Unite nella Dichiarazione su una cultura della pace rimarcano l'esigenza di promuovere il rispetto dei trattati internazionali e del diritto internazionale.

In questo caso non esistono soluzioni giuste o sbagliate a priori, soltanto dalla pratica si vedrà quale sarà la migliore soluzione, anche operare su multilivelli, adottando diverse ipotesi di soluzioni potrebbe essere efficace, con l'auspicio che l'umanità ripudierà sempre più le guerre e cercherà soluzioni pacifiche alle controversie internazionali.

\title{
CAN INTERNATIONAL LAW PREVENT WARS?
}

\begin{abstract}
In this paper, the author analyses the question whether International Law can prevent wars. International Law has always sought to resolve disputes between States peacefully. The push towards this purpose is felt mainly after the great war conflicts. The League of Nations was born according to this peacekeeping intent. Even if it is considered as a failure, the author believes that it laid the foundations for subsequent progress. Important steps are taken by the United Nations, which seeks to safeguard peace through the use of measures with or without the use of force. The author examines both of them and concludes that also this approach seems not to be entirely effective. He examines the concept of just peace and the right of peoples to peace. Finally, the focus is on the most recent trend towards the promotion of education to the culture of peace.
\end{abstract}

Keywords: Peace. International Law. Right of peoples to peace. Culture of peace.

\section{¿EL DERECHO INTERNACIONAL PUEDE IMPEDIR LAS GUERRAS?}

\section{RESUMEN}

En este artículo, el autor comienza su análisis a partir de la pregunta de si el derecho 
internacional podría impedir las guerras. El derecho internacional siempre ha tratado de resolver las disputas entre los Estados pacíficamente. El impulso hacia este propósito se siente en mayor medida después de los grandes conflictos bélicos. La Sociedad de Naciones ve su nacimiento de acuerdo con este intento de pacificación. Incluso si se define como un fracaso rotundo, el autor cree que ella ha puesto las bases para futuros progresos. Las Naciones Unidas toman herramientas importantes, que buscan salvaguardar la paz mediante el uso de medidas que incluyen el uso de la fuerza y medidas que no contemplan el uso de la fuerza. En particular, el autor examina ambos tipos de instrumentos y concluye que este enfoque no parece ser completamente efectivo. Él examina el concepto de paz justa y el derecho de los pueblos a la paz. En conclusión, la atención se centra en la tendencia más reciente a la promoción de la educación a la cultura de la paz.

Palabras clave: Paz. Derecho Internacional. Derecho de los pueblos a la paz. Cultura de la paz.

1 Orlando $(2014$, p. 11) a questo proposito ricorda quanto fosse importante per i regnanti il senso dell'onore quando si impegnavano al rispetto di un accordo: "un grande Re, proprio nel secolo XVII, Guglielmo d'Orange, portava fieramente nel suo stemma il motto: "Je maintiendrai". Il che, sia detto di passaggio, se poteva essere un motivo di orgoglio per lui, non era troppo cortese verso i suoi colleghi”.

2 Non può essere dimenticato che a Thomas Woodrow Wilson nel 1919, in quanto promotore della Lega delle Nazioni, viene attribuito il premio Nobel per la pace.

3 Ad esempio nell'Assemblea Generale le decisioni sulla sicurezza e la pace internazionale, l'ammissione di nuovi membri e il bilancio delle Nazioni Unite vengono adottate con la maggioranza dei due terzi dei presenti votanti, le altre a maggioranza semplice.

4 L'articolo 53 della Convenzione di Vienna del 1969 sul diritto dei trattati afferma: "è nullo qualsiasi trattato che, al momento della sua conclusione, sia in contrasto con una norma imperativa di diritto internazionale generale. Ai fini della presente convenzione, per norma imperativa di diritto internazionale generale si intende una norma che sia stata accettata e riconosciuta dalla Comunità internazionale degli Stati nel suo insieme in quanto norma alla quale non è permessa alcuna deroga e che non può essere modificata che da una nuova norma di diritto internazionale generale avente lo stesso carattere." (VIENNA, 1969, in linea).

5 Bobbio (2003, p. 551) rimarca le parole abbastanza forti dell'osservazione di Niccolò Machiavelli dove quest'ultimo osserva che i principi che non hanno rispettato la parola data hanno fatto grandi cose. Infatti, scrive Machiavelli (1913, p. 102; 2009, p. 56): “non di manco si vede, per esperienzia ne' nostri tempi quelli principi avere fatto gran cose che della fede hanno tenuto poco conto, e che hanno saputo con l'astuzia aggirare e' cervelli delli uomini; et alla fine hanno superato quelli che si sono fondati in sulla lealtà.”

6 Tra le teorie che ritengono giusto l'attacco preventivo c'è quella di Hobbes (2000), mentre Hugo Grotius, Christian Wolff e Emmeric de Vattel lo reputano generalmente illegittimo o sotto parametri molto ristretti (VIOLA, 2005, p. 58).

7 Tra queste teorie vanno ricordate quelle di Hugo Grotius e Francisco de Vitoria e il principio del "vim vi repellere licet" (VIOLA, 2005, p. 57-58).

8 Viola (2005, p. 59-60) ritiene che le guerre secondo Tommaso d'Aquino devono rispondere alla richiesta di giustizia ed hanno carattere di sanzione, ovviamente in risposta a gravi ingiustizie.

9 Il rischio dello sconfinamento nella ingerenza nelle politiche nazionali altrui in Paesi che non presentano gli stessi valori culturali è un tema attuale e rilevato da diversi autori, tra cui Viola (2005, p. 61).

10 Potrebbe essere, secondo Viola (2005, p. 56), più propriamente un'operazione di polizia internazionale.

11 Su tale punto, quando si parla di "guerra giusta", ci si trova dinnanzi a quello che Viola (2005, p. 56) definisce un "ossimoro". 
12 Viola (2005, p. 49) ritiene che in questa visione il concetto di pace e quello di sicurezza siano sovrapponibili.

13 A parere di Viola (2005, p. 52-53) si è rinunciato al concetto tecnico-giuridico negativo di pace per quello più in auge nella comunità internazionale della "pace giusta".

14 Queste teorie rievocano alla mente l'idea, di vecchia memoria europea, della pax romana, cioè quella situazione di "pace" nei territori variegati racchiusi all'interno dell'impero romano.

\section{BIBLIOGRAFIA}

ARCHIBUGI, Daniele. L'utopia della pace perpetua. Democrazia e diritto, v. 32, n. 1, p. 349-378, 1992.

BOBBIO, Norberto. La idea de la paz y el pacifismo. In: BOBBIO, N. (Org.). El problema de la guerra y las vías de la paz. Tradução Jorge Binaghi. Barcelona: Ediciones Altaya. S. A., 1998. p. 157-186. 1999.

. Teoria generale della politica. A cura di Michelangelo Bovero. Torino: Einaudi,

Teoría general de la política. Edición de Michelangelo Bovero. Tradução Antonio de Cabo de la Vega e Gerardo Pisarello Prados. Madrid: Editorial Trotta, S. A., 2003.

BOTTARO, Giuseppe. The world must be made safe for democracy. Woodrow Wilson e la Prima guerra mondiale. Politics. Rivista di Studi Politici, n. 2, p. 1-19, 2014. Disponível em: <www.rivistapolitics.files.wordpress.com/2015/03/politics_2014_02_01_19_bottaro. pdf>. Acesso em: 12 out. 2016.

CAPECCHI, Gabriele; PARISI, Nicoletta; SANTINI, Andrea. L'organizzazione delle Nazioni Unite. LIUC Papers, n. 102, p. 1-44, 2002, Suppl. a marzo 2002 - Serie Impresa e Istituzioni, 18. Disponível em: <www.biblio.liuc.it/liucpapersita.asp?codice=105>. Acesso em: 12 out. 2016.

CARTA Delle Nazioni Unite. Firmata da 51 membri originari ed adottata per acclamazione a S. Francisco il 26 giugno 1945. Disponível em: <http://files.studiperlapace.it/spp_zfiles/ docs/onucarta.pdf>. Acesso em: 9 out. 2016.

CASTELLI, Alberto. Pace e guerra nel pensiero di Max Scheler. Politics. Rivista di Studi Politici, n. 2, p. 21-44, 2014. Disponível em: <www.rivistapolitics.files.wordpress. com/2015/03/politics_2014_02_21_44_castelli.pdf>. Acesso em: 12 out. 2016.

CENZI, P. Tito S.; BELLONI, P. Angelo Z. (Eds.). Summa Theologiae,1265-1274. Beato Angelico: San Tommaso d'Aquino. Disponível em: <www.aisum.it/sito/content/tommasodaquino-opere>. Acesso em: 18 out. 2016.

DE VATTEL, Emer [Emmeric]. The Law of Nations [or Principles of the Law of Nature applied to the conduct and affairs of Nations and Sovereigns]. Philadelphia: T. \& J. W. Johnson, Law Booksellers, 1844 [1797]. 
DE VITORIA, Francisco. Relectiones theologicae. Lvgdvni: Expensis Petri Landry, 1586.

Relecciones sobre los indios y el derecho de guerra. 3. ed. Madrid: EspasaCalpe, S. A., 1975.

FOCARELLI, Carlo. Lezioni di diritto internazionale. Prassi. Padova: Cedam, 2008. v. 2. GRANJA CASTRO, Dulce María; CHARPENEL ELORDUY, Eduardo. El ideal de la paz perpetua en Rousseau y Kant. Signos Filosóficos, v. 26, n. 31, p. 37-62, 2014.

GROSSO, Enrico. Democrazia rappresentativa e democrazia diretta nel pensiero di Norberto Bobbio. Rivista AIC, n. 4, p. 1-19, 2015. Disponível em: <www.rivistaaic.it/breviconsiderazioni-su-efficienza-del-governo-e-riforme-costituzionali-0d5.html>. Acesso em: 8 out. 2016.

GROTIUS, Hugo. De jure belli ac pacis. Libri Tres. Tradução Francis W. Kelsey. Oxford: Clarendon Press / London, Humphrey Milford, 1925 [1646]. v. 2.

HIPPONENSIS, Augustinus [Sant'Agostino d'Ippona]. Opera Omnia. Disponível em: <www.augustinus.it/italiano/index.htm>. Acesso em: 29 abr. 2018.

HOBBES, Thomas. De Cive. 2000. Disponível em: <www.unilibrary.com/ebooks/ Hobbes,\%20Thomas\%20-\%20De\%20Cive.pdf>. Acesso em: 18 out. 2016.

Leviathan [or the Matter, Forme, \& Power of a Common-wealth Ecclesiasticall and Civill]. London: Andrew Crooke at the Green Dragon in St. Pauls Church-yard, 1651.

KANT, Immanuel. Zum ewigen Frieden: ein philosophischer Entwurf. Königsberg: Friedrich Nicolovius, 1796 [1795].

Sobre la paz perpetua. Tradução Joaquín Abellan. 6. ed. Madrid: Editorial Tecnos, S. A., 1998.

KÜNTAY, Burak. Peace through Institutions: Woodrow Wilson and the Paris Peace Conference [Kurumlar Yoluyla Barı!: Woodrow Wilson ve Paris Barı Konferansı]. Gazi Akademik Bakı, v. 8, n. 15, p. 73-89, 2014. Disponível em: <www.gaziakademikbakis. com/index.php/gab/article/download/236/210>. Acesso em: 29 abr. 2018.

LATTANZI, Flavia. Garanzie dei diritti dell'uomo nel diritto internazionale generale. Milano: Giuffrè, 1983.

MACHIAVELLI, Niccolò. Il Principe. Con commento storico filologico stilistico a cura di Giuseppe Lisio. Firenze: G. C. Sansoni, Editore, 1913.

Il Principe. Editado por Sálvio Marcelo Soares. Meta-Libri, 1 mar. 2009 [1532], v1.1p. Disponível em: 〈www.ibiblio.org/ml/libri/m/MachiavelliNB_IlPrincipe_p.pdf>. Acesso em: 30 abr. 2018.

ORLANDO, Vittorio Emanuele. La crisi del Diritto internazionale. Annuario dell'anno accademico 1948-1949, DCXLVI dalla fondazione. Roma: Università degli studi di 
Roma, 1951, p. 16-60. Nomos: le attualità nel diritto, n. 1, p. 1-30, 2014. Disponível em: <www.nomos-leattualitaneldiritto.it/wp-content/uploads/2014/07/Nomos12014_ Documentazione.pdf>. Acesso em: 7 out. 2016.

PASTORE, Baldassare. Il diritto internazionale in un mondo in trasformazione: verso un diritto giurisprudenziale? Ars interpretandi, Giustizia internazionale e interpretazione, p. 157-193, 2001. Disponível em: <www.arsinterpretandi.it//wp-content/uploads/2001/ att_pastore.pdf>. Acesso em: 30 abr. 2018.

PIVA, Daniele. Le sanzioni nello Statuto della Corte Penale Internazionale. Zeitschrift für Internationale Strafrechtsdogmatik, n. 3, p. 141-152, 2008. Disponível em: <www.zisonline.com/dat/artikel/2008_3_221.pdf>. Acesso em: 9 out. 2016.

RECCHIA, Stefano. Kant, la pace democratica e la governante mondiale federale. In: MAFFETTONE, Sebastiano; PELLEGRINO, Gianfranco (Org.). Etica delle relazioni internazionali. Cosenza: Marco Editore, 2004. p. 371-392.

SCHUKER, Stephen A. The 1919 Peace Settlement: A Subaltern View. Reviews in American History, v. 36, n. 4, p. 575-585, 2008.

SENESE, Salvatore. Globalizzazione e diritti umani. Questione giustizia, n. 4, p. 133-145, 2010.

. Corte costituzionale e sovranità. Questione giustizia, n. 1, p. 51-56, 2015.

Disponível em: <http://www.questionegiustizia.it/rivista/pdf/QG_2015-1_10.pdf>. Acesso em: 30 abr. 2018.

TANZI, Attila. Introduzione al diritto internazionale contemporaneo. 4. ed. Padova: Cedam, 2013.

TRACHTENBERG, Marc. Reparation at the Paris Peace Conference. The Journal of Modern History, v. 51, n. 1, p. 24-55, mar. 1979.

VIENNA. Convenzione di Vienna sul diritto dei trattati. Conclusa a Vienna il 23 maggio 1969. Disponível em: <https://www.admin.ch/opc/it/classified-compilati on/19690099/201502240000/0.111.pdf>. Acesso em: 9 out. 2016.

VIOLA, Francesco. La teoria della guerra giusta e i diritti umani. In: SEMPLICI, S. (Org.). Pace, sicurezza, diritti umani. Padova: Messaggero, 2005. p. 39-68.

WILSON, Thomas Woodrow. Fourteen points: delivered in Joint Session by President Woodrow Wilson, January 8, 1918. Exodus Books. Disponível em: <www.exodusbooks. com/Samples/VP/O3FourteenPoints.pdf>. Acesso em: 30 abr. 2018.

WOLFF, Christian. Jus gentium methodo scientifica pertractatum. v. 1. Oxford: Clarendon Press / London: Humphrey Milford, 1934 [1764]. 